\title{
Fuzzy Classification Based Driving Distance Estimation for Electric Vehicles
}

\author{
C Chellaswamy, ${ }^{1, *}$, T S Geetha ${ }^{2}$, G Kannan $^{3}$ and A Vanathi ${ }^{4}$ \\ ${ }^{I}$ Department of Electronics and Communication Engineering, Lords Institute of Engineering and \\ Technology, Hyderabad, India \\ ${ }^{2}$ Department of Electronics and Communication Engineering, Sriram Engineering College, Veppampattu, \\ Chennai, India \\ ${ }^{3}$ Department of Electronics and Communication Engineering, Narayana Engineering College, \\ Andrapradesh, India \\ ${ }^{4}$ Department of Electronics and Communication Engineering, Rajalakshmi Institute of Technology, \\ Chennai, India
}

('Corresponding author's e-mail: chella_info@yahoo.co.in)

Received: 2 June 2020, Revised: 29 April 2021, Accepted: 5 May 2021

\begin{abstract}
Electric vehicle technology is an essential research field for improving full-electric vehicle (FEVs) capabilities. Different subsystem parameters in the FEVs should be monitored on a regular basis. The better these subsystems are used, the better the FEVs' performance, life, and range become. Nowadays, estimation of the state of charge (SoC) of the batteries and the driving distance is the area not been standardized sufficiently. In this study, a novel fuzzy classification method (FCM) is proposed to make the exact driving distance estimation of FEVs. The proposed FCM considers the consumed power and parameters of the battery under dynamic conditions. A test location was selected for the proposed FCM and tested under 3 different test conditions, namely, no-load, half-load and full-load conditions. Also, the performance of FCM is studied under several slope conditions, and the result shows that if the battery voltage decreases then the power consumed by the vehicle is improved in the uphill travel and the battery voltage is normal and the power consumption of the vehicle is decreased in the downhill drive. Finally, the drive distance of the proposed FCM is determined.
\end{abstract}

Keywords: Driving distance, Full electric vehicle, Fuzzy classification method, Power consumption, Travel distance estimation

\section{Introduction}

A new energy generation method is demanded due to the usage of fossil fuels in the transportation sector. The transportation sector consumed 93 million barrels of oil every day worldwide. From the total greenhouse gas emission, the transportation sector produces $27 \%$ [1]. The electric vehicle (EV) is an alternate solution for reducing harmful emissions. Both the fuel crisis and environmental pollution are 2 undividable problems. The utilization of electric vehicles saves the environment and solve the fuel crisis $[2,3]$. Nowadays, electric vehicle technology is an important research area, accepted by many countries for reduction of emission and energy saving. It also leads to the production of new vehicles [4].

EVs are a combination of electrical and mechanical systems that have different subsystems for proper operation. The subsystem includes a battery control unit, motor control unit, sensing, and monitoring unit, and communication unit for efficiently handling different functionalities [5,6]. EVs are classified as follows: 1) Plug-in Hybrid Electric Vehicles (PHEVs) and 2) Battery Electric Vehicles (BEVs). BEVs can be categorized based on various energy sources utilized [7]. PHEVs used electric motors and internal combustion engines whereas BEVs used only electric motors for the entire drive system. Various power sources such as fuel cells, batteries, supercapacitors, and solar panels are used as hybrid or separate [8]. An automatic recharging mechanism based on renewable energy has been proposed by Chellaswamy. Both solar and wind energy remain used for charging the battery packs of the electric vehicle [9]. The battery packs connected in the BEV give the required energy for the drive system. The battery packs of EVs are charging through park stations, the standard home outlets, and 
roadside units, etc. Recharging the storage system present in EVs requires a couple of hours, and the capacity of the battery pack defines the charging time [10,11].

The energy stored in the battery pack defines the distance travelled by the vehicle. The drive distance is estimated using various ways, namely, some deterministic methods, the standards created by the Environmental Protection Agency (EPA), and the measured data of previous trips. However, these methods do not consider dynamically changing environmental conditions which are affecting the vehicle. As a result, the accuracy of those methods is very less [12]. There are 2 different approaches are followed to estimate the driving distance of EVs. The $1^{\text {st }}$ is a model-based approach and the $2^{\text {nd }}$ one is the historical knowledge-based approach. The knowledge-based approach does not include a model instead it uses previous battery consumption data and driving information of the vehicle. As a result, the accuracy of the knowledge-based approach is less [13]. Daina et al. [14] suggested a model-based drive distance estimation method that includes the mathematical model of all the subsystems of the vehicle. This is the reason for the accuracy of the model-based approach is higher than the prediction method. The modelbased approach requires a high-precision mathematical model for providing an accurate result [15]. On the other hand, the combination of both the model-based approach and the knowledge-based approach is used in a hybrid vehicle. The model used a multivariate regression model for evaluating the measured data [16]. A novel method is introduced to estimate the drive distance of the electric vehicle using different parameters such as temperature, battery capacity, battery discharge voltage, available energy in the storage system, and the energy consumption of the vehicle. The linear regression method includes driver behavior in real road driving and the parameters of the vehicle are considered dynamically for the analysis. Even though this method provides a better result, the sensitivity is easily affected by variation in the driving conditions $[17,18]$.

The usage of EVs is affected by various factors such as longer battery charging time and limited battery capacity. So that the EV consumers and manufacturers meeting certain concerns. Hence it is important to know the existing charge of the battery and the corresponding coverage distance [19]. For estimating the drive distance of the $\mathrm{EV}$, the $\mathrm{SoC}$ information is required. Tannahill et al. [20] proposed a low-cost microcontroller-based SoC estimation method for calculating the drive distance of the vehicle. The variation of $\mathrm{SoC}$ is taken into account and the corresponding distance is estimated and informs the driver. Boyraz and Dogan [21] developed a hybrid system using fuzzy logic and an artificial neural network (ANN) for vehicle classification during traffic. The fuzzy logic control is used to identify the number of vehicles through the vehicle acoustic signal in the traffic junction and fix the lighting time of different signals. Dawei et al. [22] proposed a neuro-fuzzy classifier and classical neural network-based sensory network for real-time emotion recognition. A webcam is used to send the video stream of a user's face identifying the emotion. For monitor and controlling different subsystems of EVs, inter-vehicle communication is essential. It can be achieved through the communication protocol called the Controller Area Network (CAN) [23]. Different parameters of the vehicle subsystem can be collected and passed the control information to the target during the operation. The other communication protocols such as Local Interconnect Network (LIN) and Media Oriented Systems Transport (MOST) are widely used for intercommunication in vehicles [24,25]. Power Line Communication Protocol (PLCP) was developed by Bassi et al. [26] for inter-vehicle communication over electric lines used in the vehicle. PLCP is widely used in vehicle subsystem communication and building automation systems.

In the above literature, a fuzzy logic-based classification method (FCM) was used in a limited number of studies on EVs. In this study, FCM is used to estimate the drive distance of EVs. The command should be transmitted and the required information should be collected without any problem. For monitoring and controlling different subsystems of EVs, a CAN inter-vehicle communication protocol is used. The EV's drive system directly affects due to the following parameters: 1) external loads acting on the vehicle, 2) traffic condition, 3) environmental factors, and 4) road type. Changes in these parameters and the status of the batteries directly affect the instantaneous power consumption of the vehicle. In this study, the driving force is estimated using 4 different parameters such as the weight force of the vehicle, the force due to vehicle inertia, wind resistance, and force of gravity of the vehicle. The instantaneous power consumption can be evaluated through the dynamic change of parameters and the status of the battery charge. For simplifying the drive distance estimation, the fuzzy classifier is proposed. The rest of the paper is structured as follows: Section 2 explains the material and methods used. Section 3 describes the Designing of the fuzzy classifier. Experimental results and discussion are given in Section 4 and the conclusion is given in Section 5.

\section{Material and methods}


In practice, various physical forces affect the drive system of EVs. Various forces affecting the drive system are illustrated in Figure 1. The drive system should have the capability to handle these forces, namely, the force due to the wind $\left(\mathrm{F}_{3}\right)$, gravitational force $\left(\mathrm{F}_{1}\right)$, the force due to inertia $\left(\mathrm{F}_{2}\right)$, and rolling resistance force $\left(\mathrm{F}_{4}\right)$. The movement of the vehicle will be affected by these dynamic parameters. The knowledge of the physical resistance of each subsystem present in the EVs affects the drive system is important for guaranteeing the movement of the vehicle. The drive system must exceed the forces due to the physical resistances for performing the movement. The driving force $\left(f_{D}\right)$ of a vehicle can be expressed as:

$f_{D}=g V_{\text {mass }} \cos (a) C_{R R}+V_{\text {mass }} A_{v}+0.5 \rho A S_{a}\left(S_{v}+W_{s}\right)^{2}+g V_{\text {mass }} \sin (a)$

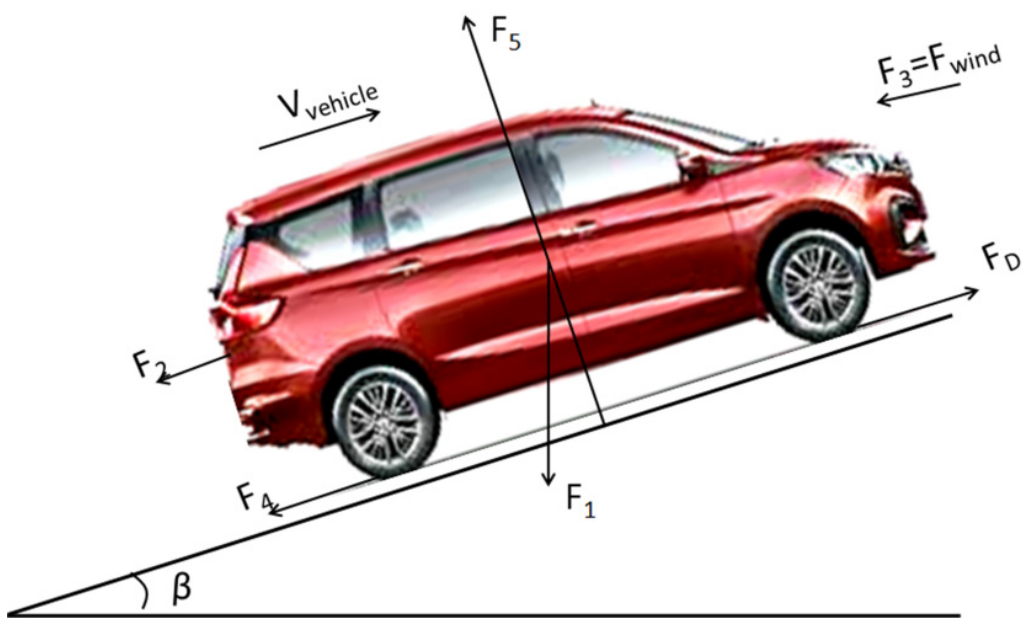

Figure 1 Vehicle affected by different forces.

In Eq. (1), the $1^{\text {st }}$ term denotes $\mathrm{F}_{4}$ (Figure 1), where CRR is the coefficient of rolling resistance $\left(C_{R R}=3.61 S_{v}\right), A_{v}$ is the vehicle acceleration $\left(\mathrm{m} / \mathrm{s}^{2}\right)$, is the road slope (degrees), $\mathrm{V}_{\text {mass }}$ is the weight of the vehicle $(\mathrm{kg}), \mathrm{g}$ is the gravitational acceleration $\left(\mathrm{m} / \mathrm{s}^{2}\right)$. The $2^{\text {nd }}$ term $\left(\mathrm{F}_{2}\right.$ in Figure 1) indicates the force due to vehicle inertia $(\mathrm{N})$, where $S_{v}$ is the speed of the vehicle in $\mathrm{km} / \mathrm{h}$. The $3^{\text {rd }}$ term $\left(\mathrm{F}_{3}\right.$ in Figure 1) indicates the force due to wind resistance, where $\rho$ is the density of air $\left(\mathrm{kg} / \mathrm{m}^{3}\right), A$ is the aerodynamic coefficient, $S_{a}$ is the surface area of the vehicle $\left(\mathrm{m}^{2}\right), \mathrm{W}_{\mathrm{s}}$ is the speed of wind $(\mathrm{m} / \mathrm{s})$, and the last term $\left(\mathrm{F}_{1}\right.$ in Figure 1) indicates the force of gravity of the vehicle $(\mathrm{N})$. In addition, $\mathrm{F}_{5}$ of Figure 1 denotes the $1^{\text {st }}$ term of Eq. (1) except CRR. Some of the vehicle parameters are changed dynamically and some of them are fixed [27]. The torque generated in a mechanical system can be expressed as:

$M_{T}=F_{v} W_{r}$

where $\mathrm{M}_{\mathrm{T}}, \mathrm{F}_{\mathrm{v}}$, and $\mathrm{W}_{\mathrm{r}}$ are the generated torque $(\mathrm{Nm})$, the Driving force of the vehicle, and the radius of the wheel $(\mathrm{m})$ respectively. The driving force of the vehicle can be expressed as based on [27] as:

$D_{P}=F_{v} S_{v}$

where $D_{p}$ represents the drive power $(W), S_{v}$ is the speed of the vehicle $(\mathrm{km} / \mathrm{h})$. The wheel torque can be expressed as:

$M_{w}=\frac{M_{T}}{2}$

where $\mathrm{M}_{\mathrm{w}}$ is the wheel torque $(\mathrm{Nm})$ and the angular speed $\left(\boldsymbol{\omega}_{w}\right)$ of the wheel can be expressed as:

$\omega_{w}=\frac{S_{v}}{M_{w}}$ 
By using the above equations, the required power for the vehicle movement can be calculated and the driving system is designed.

\section{Proposed approach}

In this study, for estimating the drive distance an EV is required. The basic mechanical, electrical and electronic system required for the EV is manufactured. The FCM was applied in the EV and the test was carried out under different conditions. The technical specification of EV is listed in Table 1. The vehicle is designed with 4 wheels with a total weight of $780 \mathrm{~kg}$ including other accessories. The batteries are connected to the test vehicle are shown in Figure 2. The motor drive system was purchased and calibrated.

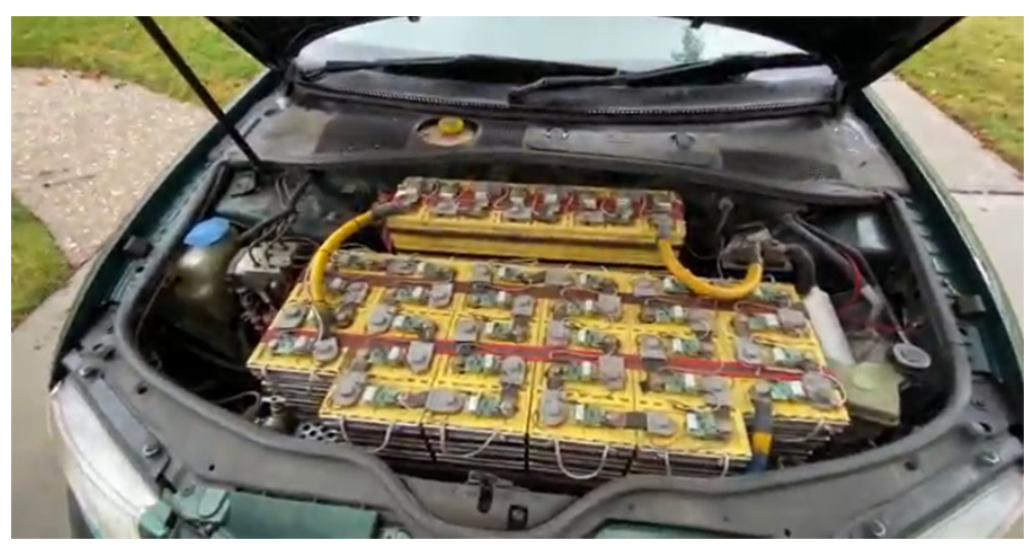

Figure 2 Battery packs are connected in the front portion of the test vehicle.

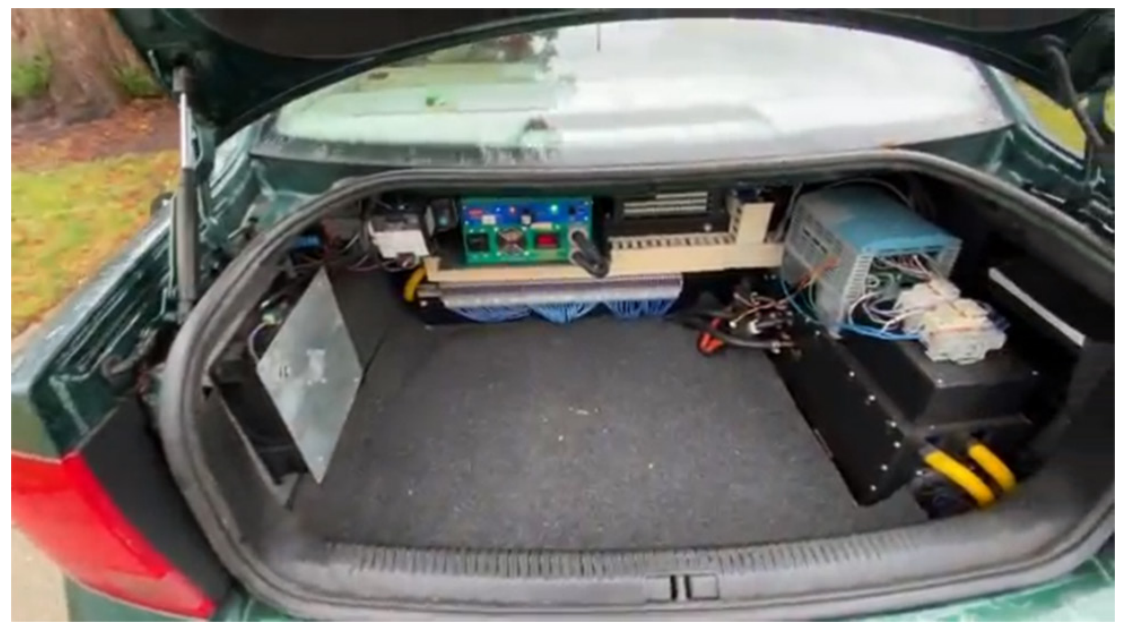

Figure 3 Battery measurement and monitoring system used in the EV.

Communication between the master controller to other subsystems present in the EV is done through the CAN protocol. The voltage sensor (LV25P) is used to measure the voltage values of batteries continuously. The battery temperature is measured using a resistance temperature detector (RTD) [17]. For measuring and monitoring the dynamic parameters of the batteries an electronic circuit has been developed. The battery measurement and monitoring system are shown in Figure 3. The current sensor LA125P was used to measure the total instantaneous current consumed by the system.

Table 1 Technical specifications of the EV under test. 


\begin{tabular}{lcc}
\hline \multicolumn{1}{c}{ Parameters } & Values & Unit \\
\hline Number of wheels & 4 & - \\
Width & 1.2 & $\mathrm{~m}$ \\
Length & 2.75 & $\mathrm{~m}$ \\
Height & 1.8 & $\mathrm{~m}$ \\
Brake system & Double rope rear braking system & Rear \\
Tyre diameter & 395 & $\mathrm{~mm}$ \\
Tyre width & 170 & $\mathrm{~mm}$ \\
Distance between wheels & 0.95 & $\mathrm{~m}$ \\
Motor controller & Sepex controller (KDZ72400) & \\
Motor type & $48 \mathrm{~V}, 100 \mathrm{~A}$ Zibo DC motor & $\mathrm{kg}$ \\
Weight of motor & 29 & $\mathrm{~kW}$ \\
Motor power & 4.8 & $\%$ \\
Efficiency of motor & 79 & \\
\hline Battery & & $12 \mathrm{~V} \times 4$ \\
\hline Type & Gel battery & Volt \\
Maximum Voltage & 12 & Wh \\
Battery power & 5200 & Volt \\
Charging cut-off voltage & 13.5 & Volt \\
Discharge cut-off voltage & 11.5 & \\
\hline
\end{tabular}

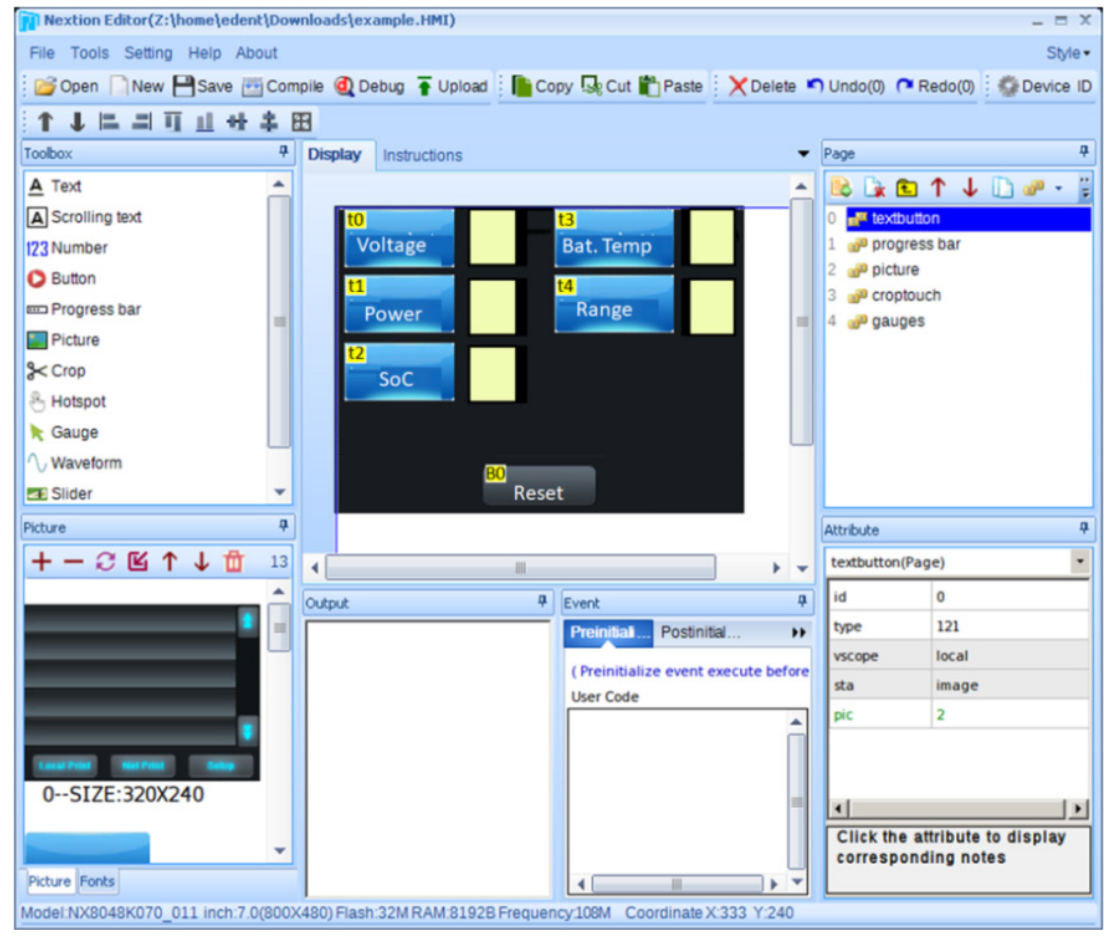

Figure 4 Nextion touch screen displays the vehicle information.

The controller (Atmega328) is used to perform control operations based on the signals received from different sensors. A touch screen display system is developed for showing various parameters; the user can control various parameters of the EV by using this. The Nextion touch screen has an ARM controller that controls the display. Both the controllers are communicated with a baud rate of 9600 . Various parameters of the vehicle that have been designed for EV are shown in Figure 4. The touch screen displays various parameters such as the speed of the vehicle, drive distance, total power, power consumption, and SoC. A proximity sensor is used to measure the speed of the vehicle which is mounted near the wheel. The wheel speed of the EV is measured through the sensorless anti-lock braking system (ABS). The back EMF of the In-Wheel motor is used to estimate the moving speed of the vehicle. 
Furthermore, the accuracy of the speed estimation is compared with an optical encoder output. The main advantages of sensorless measurement include a reduced number of connections, decreased maintenance, easier miniaturization of the system, and lower system cost.

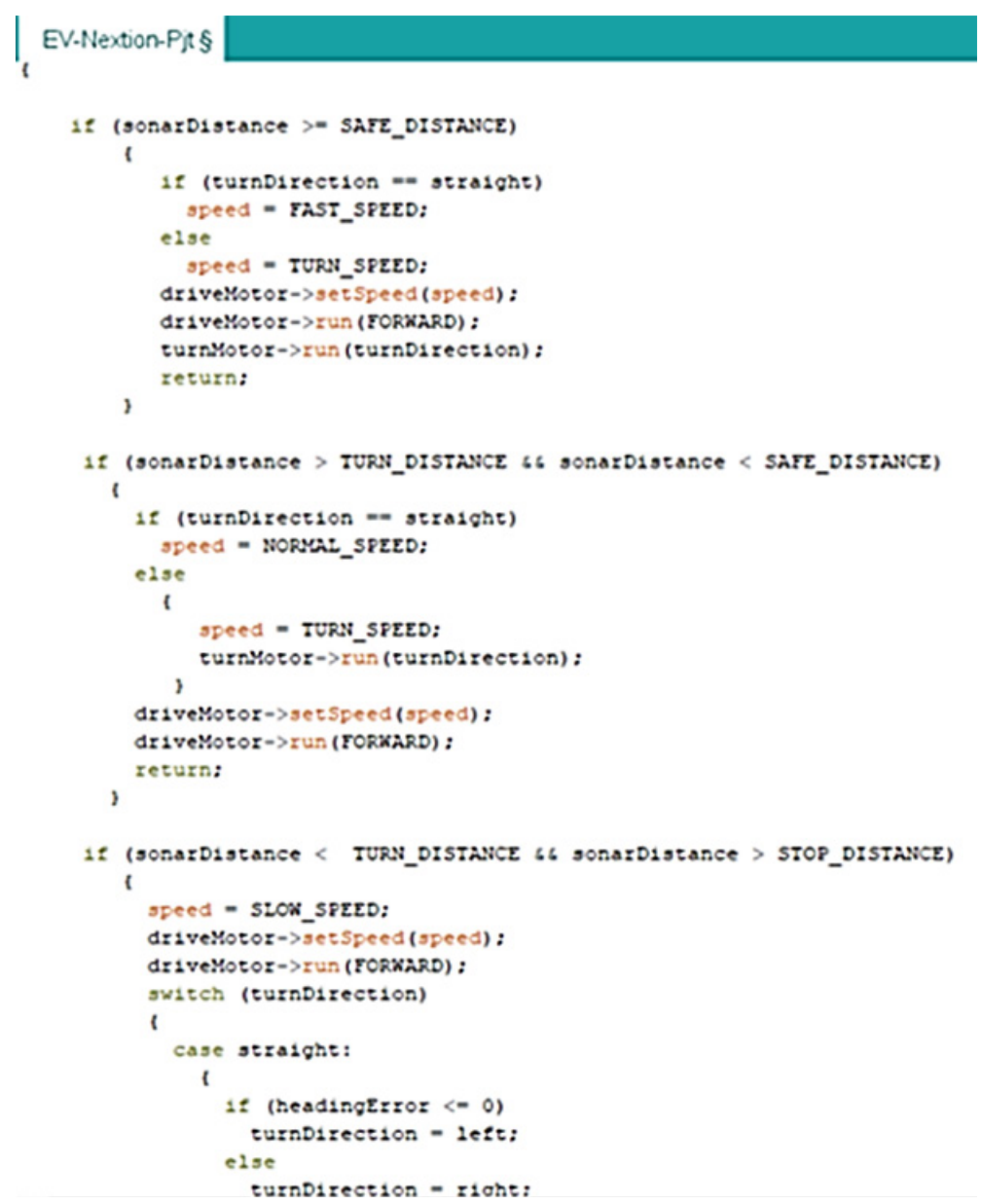

Figure 5 Snapshot of the master program control (MPC) using Arduino UNO.

\section{Design of touch screen}

Nextion display is a Graphical User Interface (GUI) mainly used in monitoring and control applications. The Nextion is a resistive touchscreen that has a built-in ARM microcontroller for controlling the display. It is used to create buttons, store images, and text-based on the requirement. Serial communication with baud at a rate of 9600 is used to communicate with other devices such as Arduino, Raspberry Pi, ESP8266, ESP32 and so on. The Nextion Editor is used to design the GUI in which one can add buttons, progress bars, gauges, and text labels for the user interface in an easier way.

In this study, we added a black background image with dimensions equal to the Nextion display. Various elements present in the toolbox of the Nextion Editor and the designed GUI for the drive distance estimation is shown in Figure 4. The size and position of the elements were defined in the attribute area. All the elements have their object name and unique identification number. The touchable component should be triggered for the specific event. The compilation was carried out and downloaded the TFT file to the corresponding work to the Nextion display.

\section{Master program control (MPC)}

The MPC has 4 main modules, namely, Li-ion battery charge control and SoC module, speed control module, power converter module, and range estimation. In addition, it also manages slave controllers. The snapshot of the MPC is shown in Figure 5. The MPC determines the cell operating conditions and maintains the SOC accordingly. Standard SOC range for Li-ion battery varies between 
$3.75-3.89 \mathrm{~V}$. If a mismatch is found in any cell, the MPC switch on the bidirectional DC-DC converter to charge the corresponding cell. The converter has both the forward and reverse converter for charging and discharging the battery. A dynamic braking system is used and a drive control is used in the EVs for controlling the speed. The MPC controls the electronic circuit that regulates the speed of an electric motor. The MPC also controls the main DC-DC converter for providing a lower DC voltage (48 V or 12 V) required for fans, window motors, wiper, interior lights, headlights, and other systems. Also, the MPC estimates drive distance based on the available energy of the battery packs. The drive distance estimation is clearly explained in section 3 .

\section{Design of fuzzy classifier}

For estimating the drive distance of EVs, FCM was used. The model-based method is included to predict the drive distance. The fuzzy classification method was chosen because it has different advantages such as it can be easily developed, it produces fast results, and higher accuracy [28]. The model of a fuzzy classification method for drive distance estimation is shown in Figure 6. Figure 6 indicates that the SoC of the battery, instantaneous power, distance measurement $\left(\mathrm{F}_{\mathrm{D}}\right)$, and speed measurement are the input to the model. During fuzzification, the set of input is decomposed into one or more fuzzy sets. The classifier output is created based on the associated rules by the inference engine. The output of the inference engine transforms the range estimation into real-world user understandable data.

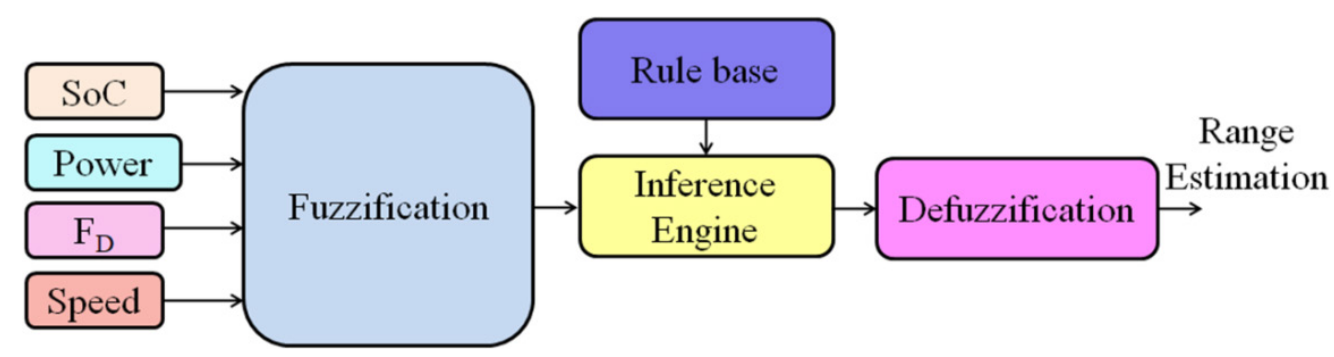

Figure 6 Model of FCM for drive distance estimation.

The membership function is used to represent the instantaneous power and SoC parameters. The parameters are measured momentarily, and the membership function values are represented between 0 1. The classifier output is produced by the inference engine of FCM, processing the rules related to the drive distance estimation from the set of rules framed. The output of the inference engine of FCM is transformed into real-world space for user understanding. The theoretical calculation was done before the experiment by considering the route and the vehicle parameter is listed in Table 2 . The torque estimated drive distance and consumed power were estimated including the road and slope parameters. Here the coefficient of friction force $(0.035)$, vehicle speed $(20 \mathrm{~km} / \mathrm{h})$, and wind speed $(5 \mathrm{~km} / \mathrm{h})$ are considered for calculation.

The calculation was performed before the experiment for different road slopes at a maximum of 8 degrees. The values are applied to Eqs. (1) - (5) and determine the parameters. After the theoretical calculations were completed the experiment was conducted in the EV. A comparison is performed with the theoretical and experimental results. It is seen that the upper limits of the estimated values should be close to the membership function of the classifier. 
Table 2 Estimated results of torque, drive distance, and consumed power before the experiment.

\begin{tabular}{ccccc}
\hline Loading & Slope of road & $\begin{array}{c}\text { Power consumed } \\
(\mathbf{W})\end{array}$ & $\begin{array}{c}\text { Torque } \\
(\mathbf{N m})\end{array}$ & $\begin{array}{c}\text { Distance } \\
(\mathbf{k m})\end{array}$ \\
\hline \multirow{3}{*}{ No-load } & 0 & 328.84 & 56.32 & 45.82 \\
& 2 & 352.40 & 61.53 & 42.05 \\
& 4 & 381.72 & 67.98 & 39.42 \\
& 6 & 407.39 & 71.25 & 36.93 \\
Medium-load & 8 & 435.81 & 76.91 & 34.71 \\
& 0 & 354.73 & 61.52 & 42.83 \\
& 2 & 389.87 & 68.03 & 39.74 \\
& 4 & 415.90 & 73.82 & 36.18 \\
Full-load & 6 & 452.74 & 78.49 & 33.79 \\
& 8 & 478.39 & 83.18 & 30.83 \\
\hline & 0 & 407.39 & 72.81 & 36.70 \\
& 4 & 447.37 & 79.67 & 34.72 \\
& 6 & 482.74 & 85.63 & 32.81 \\
& 8 & 519.31 & 92.06 & 30.68 \\
& 5 & 556.84 & 99.18 & 27.48 \\
\hline
\end{tabular}

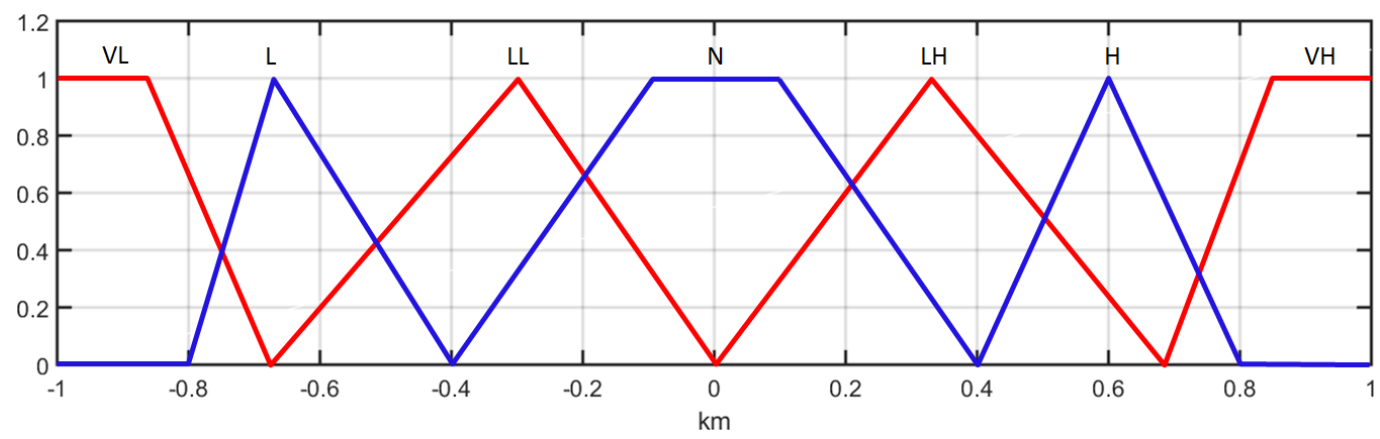

Figure 7 Output membership functions of the classifier.

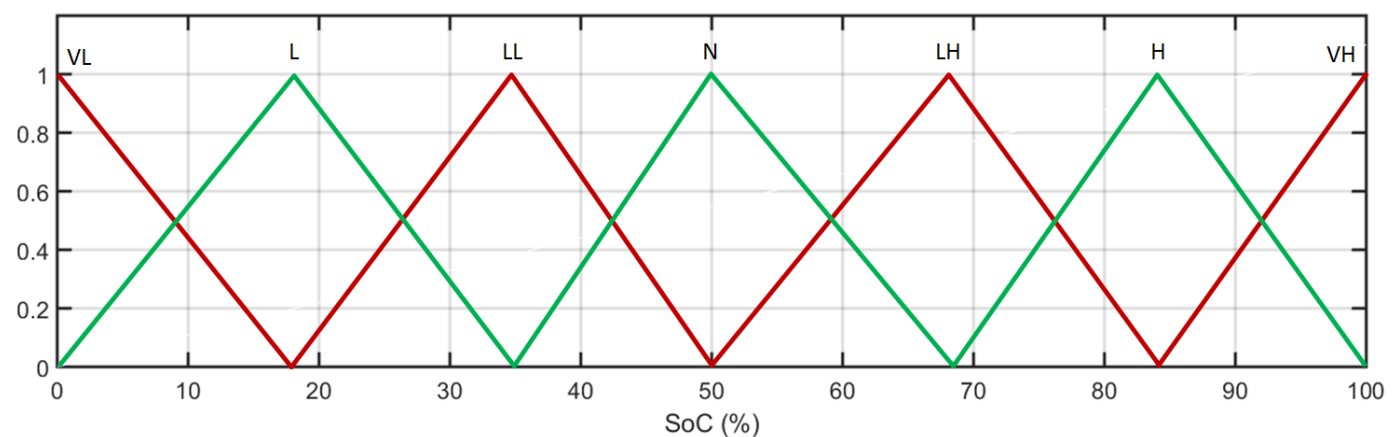

Figure 8 Membership functions of the battery charging status.

The output membership function of the classifier is shown in Figure 7. The values are selected between -1 and 1 for estimating the drive distance [29]. The drive distance was estimated for EV by evaluating the variables under running conditions. The power consumption and SoC of the battery are taken as input variables of the FCM, which has 7 membership functions.

The membership functions (input and output) of FCM are linguistically labelled as very very low (VVL), very low (VL), low (L), little low (LL), normal (N), little high (LH), high (H), very high (VH), and very very high (VVH) [30]. The membership functions of the charging status of the battery are shown in Figure 8. SoC is articulated as $0-100 \%$. 


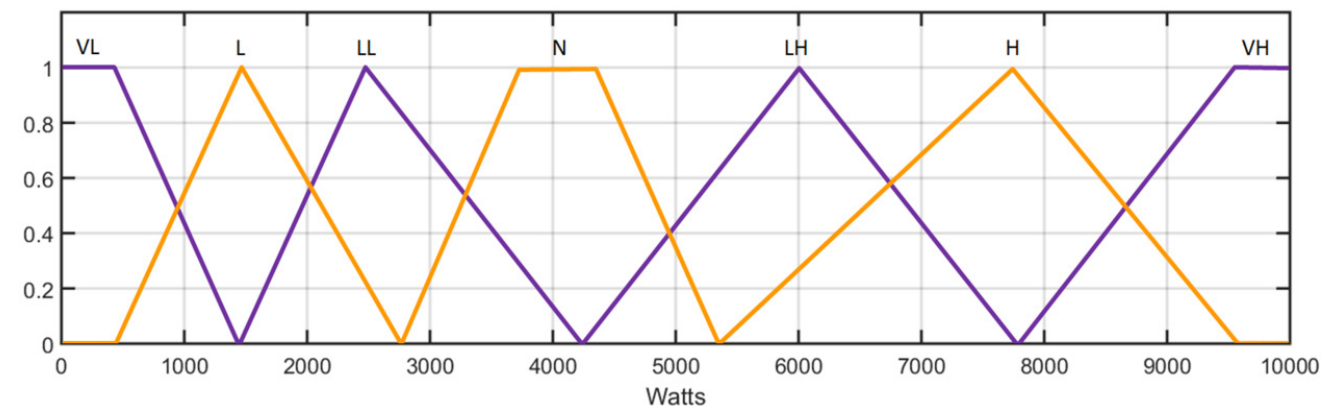

Figure 9 Membership functions of instantaneous power.

Table 3 Rule base for the proposed FCM.

\begin{tabular}{cccccccc}
\cline { 1 - 1 } SoC & \multirow{2}{*}{ VL } & \multirow{2}{*}{ L } & \multirow{2}{*}{ LL } & N & LH & H & \multirow{2}{*}{ VH } \\
\cline { 1 - 5 } VL & N & L & L & VL & VL & VL & VL \\
L & N & LL & L & L & L & VL & VL \\
LL & N & LL & LL & L & L & VL & VL \\
N & N & N & LL & LL & L & L & VL \\
LH & N & N & N & LL & LL & LH & L \\
H & N & VH & H & LH & N & LL & L \\
VH & N & VH & VH & H & LH & LH & N \\
\hline
\end{tabular}

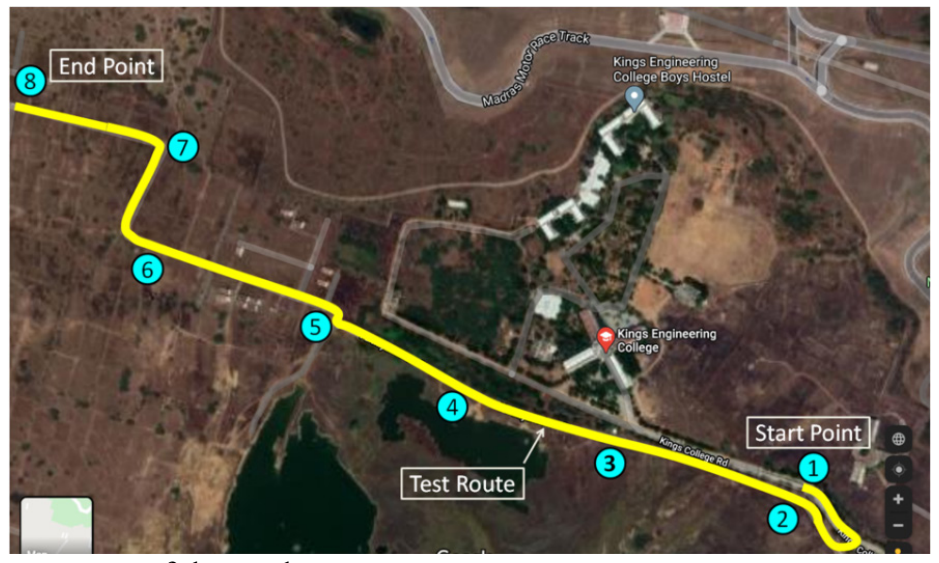

Figure 10 Selected test route of the study.

The membership functions of the instantaneous power ( $2^{\text {nd }}$ input) of FCM is shown in Figure 9. Both the trapezoidal and triangular membership functions are used in Figures $\mathbf{7}$ and $\mathbf{8}$, respectively. The rule base is created according to the input variables of the membership functions of the FCM for drive distance estimation. The rule base for the proposed FCM is listed in Table 3. The theoretical calculations and expert opinion have been considered for constructing the rule base of the proposed FCM.

\section{Results and discussion}

\section{Experimental results}

The theoretical calculations such as torque, power, and distance were made before the experiment. A test route was selected in Chennai city and the test was carried out for 3 different scenarios such as noload, half loaded, and fully loaded. Eight test points (A-H) have been fixed in the test area is shown in Figure 10. Distance between the test point $A$ to $H$ is $2.1 \mathrm{~km}$ with a different slope was used in the experiment. Before starting the drive distance estimation, the communication protocol was verified. The drive distance is estimated for all the test conditions and the dynamic parameter of the vehicle is updated 
simultaneously. The batteries of the vehicle are fully charged and the test was carried out. The measured output is displayed using the Nextion NX3224T024 HMI TFT LCD. The driver is taken into account at no-load condition.

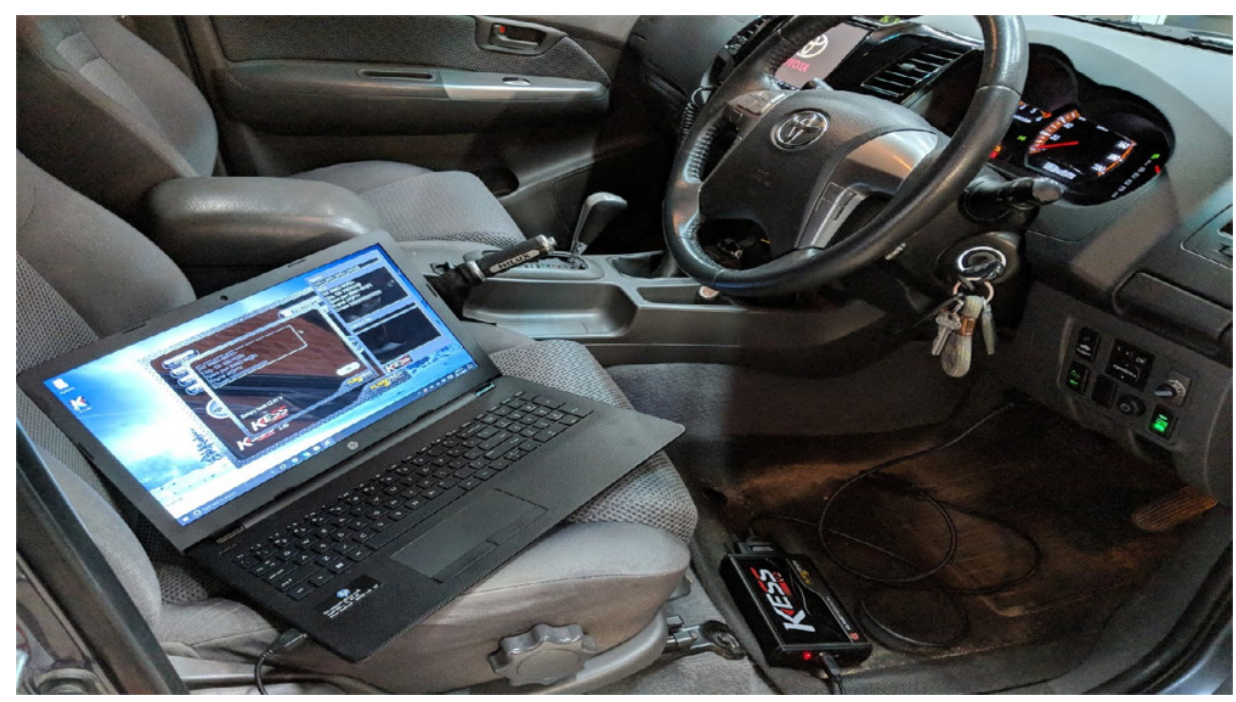

Figure 11 CAN protocol verification using Picoscope.

\section{CAN communication test}

The CAN communication was tested before the drive distance estimation. It is necessary to know the communication between different subsystems of EV through the CAN bus. It is known that the voltage between the CAN-low line to the CAN-high line should be $2.5 \mathrm{~V}$ and the equivalent resistance between the communication line should be $60 \Omega[31,32]$. The EV was powered and the subsystem of EV connected using CAN protocol was observed through the Picoscope 4000 series is shown in Figure 11. This software is used for real-time signal acquisition supported on Microsoft Windows, Mac OS X, Ubuntu, and Debian platforms. The main purpose of Picoscope is to analyze and view real-time signals from data loggers and oscilloscopes.

Various parameters of EV are sent via CAN network will be identified using message IDs. For example, the motor current, SoC information, and battery voltage were used with the ID numbers $0 \times 1$, $0 \times 5$ and $0 \times 2$, respectively. The CAN test device is connected to the vehicle and the subsystem connection is shown in Figure 11. After the connection was established, the CAN protocol was analyzed using the Picoscope software 4000 series. The ID number, the data, length of the data, and CRC (cyclic redundancy check) bit of each data stream were analyzed. The measured signal for the initial communication between all the subsystems was carried out and the communication between the CAN protocol and the motor controller is shown in Figure 12.

In Figure 12, $\mathrm{A}$ is the start of the message signal, $\mathrm{B}$ is the message ID, $\mathrm{C}$ is the data length code (DLC) signal, D is the CRC calculated field, E is the CRC field decoded, and F is the processing time. From Figure 12 it is observed that the potential difference between CAN terminals high and low in the physical layer is 0 volt. At the start of the message, the potential difference between CAN high and low terminals is 5 volts. When the message identification code is identified by the hardware, the potential difference between CAN-low to CAN-high is probably 0. The DLC indicates the length information of the message, in a standard, CAN message 4-bit length is used for the message. In Figure 12, the portion $\mathrm{D}$ and $\mathrm{E}$ shows the cyclic redundancy check (CRC), which indicates the accuracy of the sending message and received by the hardware. The length of the field is limited to 16-bits. The CRC code is sent along with the message. The message is decoded by the receiver and matches the CRC processed by the receiver. If any mismatch is identified, the data is invalid. The last portion in Figure 12 is F. It indicates the end of the delimiter. The measured output shows that communication is successful between the CAN to the master controller. 


\section{Drive distance estimation}

Various parameters used to estimate the drive distance of EV is listed in Table 4. Distance between different test points and the corresponding slope is given in Table 4. It is understood that the EV climbed initially and maintain up to 5 and downhill between 5 to finish point. To make the analysis easier, the total driving distance is divided into different sections. The EV starts from point 1, passes through 2, 3, 4, $5,6,7$ and ends with point 8 .

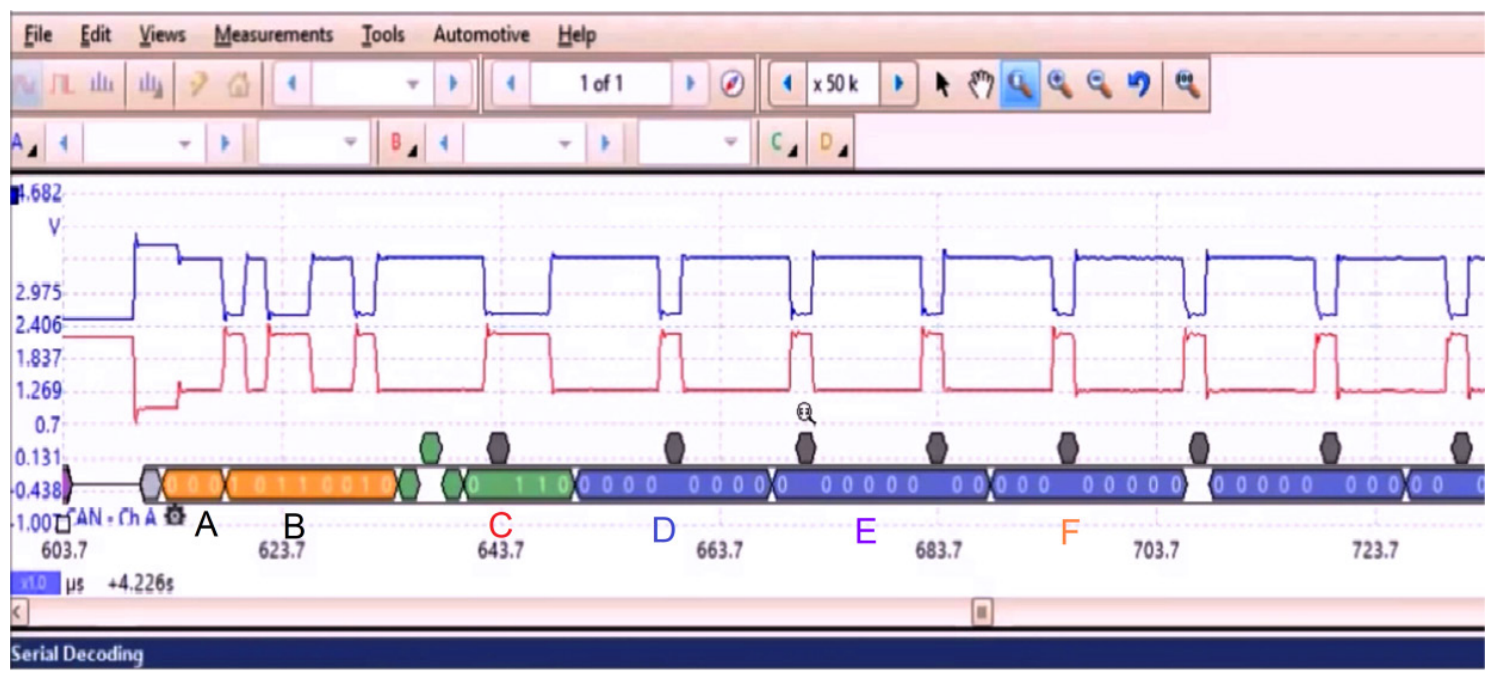

Figure 12 Analysis of CAN protocol for the proposed distance estimation.

Table 4 Distance and slope used to estimate drive distance of EV.

\begin{tabular}{lccccccc}
\hline & $\mathbf{1 - 2}$ & $\mathbf{2 - 3}$ & $\mathbf{3 - 4}$ & $\mathbf{4 - 5}$ & $\mathbf{5 - 6}$ & $\mathbf{6 - 7}$ & $\mathbf{7 - 8}$ \\
\hline Distance (m) & 402.30 & 309.45 & 294.29 & 217.23 & 390.36 & 280.96 & 205.45 \\
$\begin{array}{l}\text { Average slope } \\
\text { (\%) }\end{array}$ & 2.48 & 2.62 & 1.94 & -2.94 & -1.73 & -2.184 & -2.74 \\
\hline
\end{tabular}

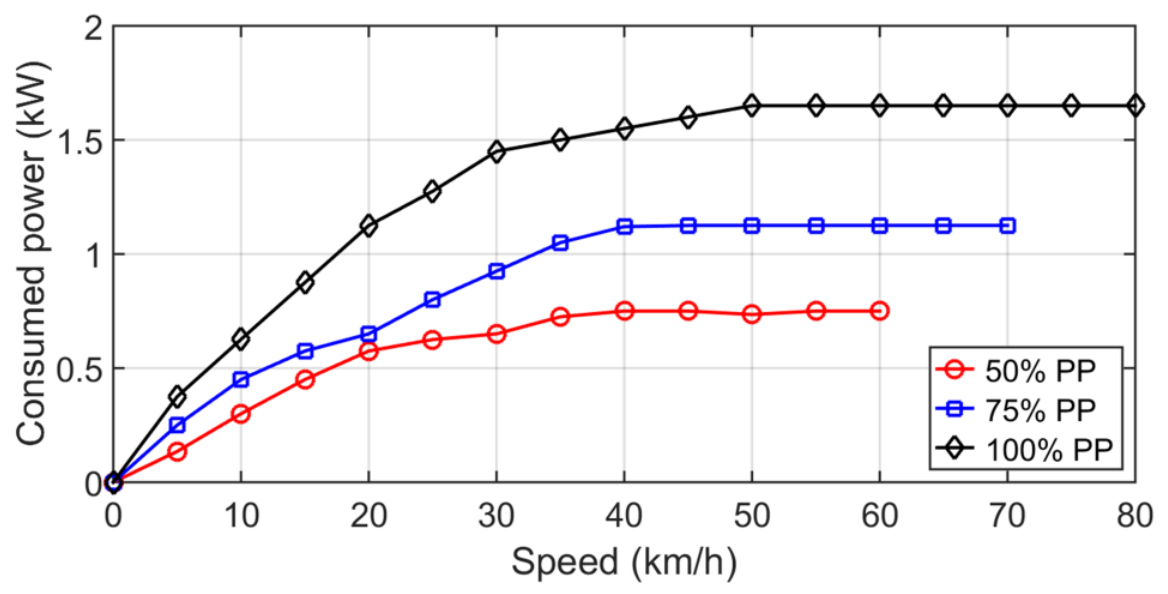

Figure 13 Power consumption of EV under different pedal position. 
Table 5 Various parameters of EV under different loading condition.

\begin{tabular}{|c|c|c|c|c|c|c|c|c|c|}
\hline \multirow[b]{2}{*}{ Loading } & \multicolumn{3}{|c|}{ Vehicle Parameters } & \multicolumn{3}{|c|}{ Motor Parameters } & \multicolumn{3}{|c|}{ Battery Parameters } \\
\hline & $\begin{array}{c}\text { Load } \\
(\mathrm{kg})\end{array}$ & $\begin{array}{c}\text { Time } \\
\text { (s) }\end{array}$ & $\begin{array}{c}\text { Average } \\
\text { speed } \\
(\mathbf{k m} / \mathbf{h})\end{array}$ & $\begin{array}{c}\text { Max. } \\
\text { current } \\
\text { (A) }\end{array}$ & $\begin{array}{c}\text { Max. } \\
\text { Torque } \\
\text { (Nm) }\end{array}$ & $\begin{array}{c}\text { Power } \\
\text { consumed } \\
(\mathbf{W h})\end{array}$ & $\begin{array}{c}\text { Average } \\
\text { current } \\
\text { (A) }\end{array}$ & $\begin{array}{c}\text { Average } \\
\text { voltage } \\
\text { (V) }\end{array}$ & $\begin{array}{c}\text { Charge } \\
\text { status } \\
(\%)\end{array}$ \\
\hline No-load & 74 & 1980 & 20.2 & 100 & 130 & 520.38 & 37.85 & 49.93 & $79.8 \%$ \\
\hline Half-load & 159 & 2700 & 16.5 & 96 & 124 & 621.47 & 48.37 & 49.18 & $76.4 \%$ \\
\hline Full-load & 312 & 3120 & 14.5 & 110 & 150 & 846.82 & 62.76 & 48.21 & $65.7 \%$ \\
\hline
\end{tabular}

Driving style also affects the power consumption of the vehicle thereby distance coverage. In this study, the power consumption of the EV under 3 different pedal positions (PP) such as 50, 75 and $100 \%$ have been considered. Different PP and the corresponding power consumption and speed are shown in Figure 13. Figure 13 indicates that higher speed is achieved for $100 \% \mathrm{PP}$ compared to lower PP. The maximum speed of the EV reduces for lower PP consequently lower acceleration. The output received by the Nextion TFT display is shown in Figure 14. The driver can understand the coverage distance of the vehicle for the charge available in the battery packs.

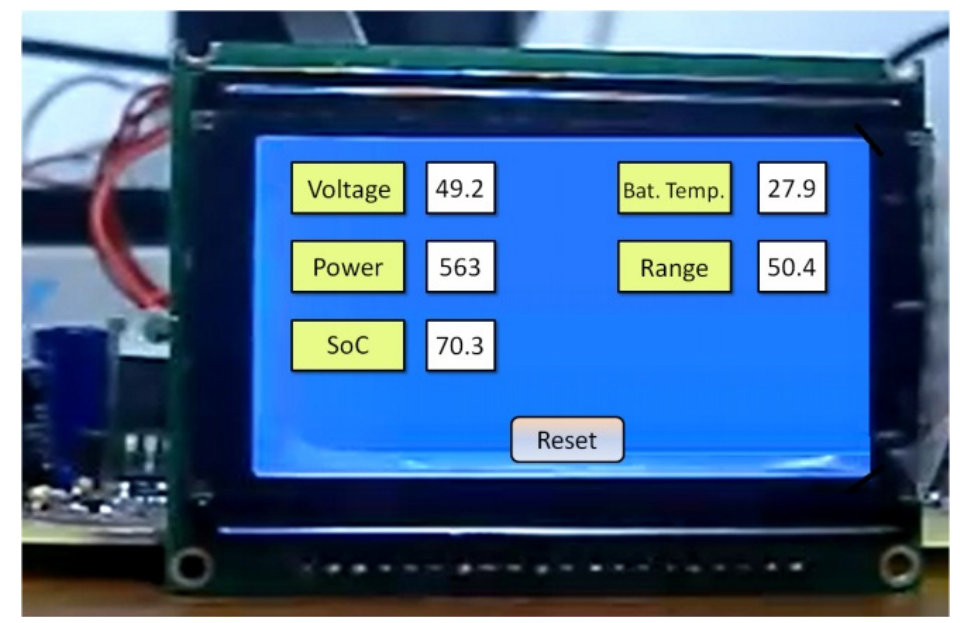

Figure 14 Measured output displayed in Nextion TFT display.

The slopes of the road directly affect the power consumption of the EV. Initially, the test was carried out with no-load condition (only with driver) for the test route, $2.1 \mathrm{~km}$ with $74 \mathrm{~kg}$ of load. The information received from the subsystem of EV and the responses of the FCM was noted. The no-load test was completed in the 1980s. During the experiment, the average voltage and current of the battery are $49.93 \mathrm{~V}$ and $37.85 \mathrm{~A}$, respectively. The power consumed by the EV was $520.38 \mathrm{Wh}$. The drive distance of the EV estimated by the proposed FCM from the test point A to G is shown in Figure 15.

The 3 different loading conditions of the EV were conducted at different times and the results of the 3 experiments are illustrated in Figure 15. The slope climbs up to point $C$ to $D$ with a slope of 2.94 and downhill starts from point $\mathrm{E}$ to the end of the driving route. The end section has a downhill slope of -2.74 (Table 3). Figure 15 it is observed that the drive distance slowly decreases at the start of $\mathrm{C}$ and it increases from D based on the output of FCM. Similarly, the drive distance of the EV estimated by the proposed FCM from the test point $G$ to $A$ is shown in Figure 16. It is observed that the power consumption is less downhill compared to uphill drive. 


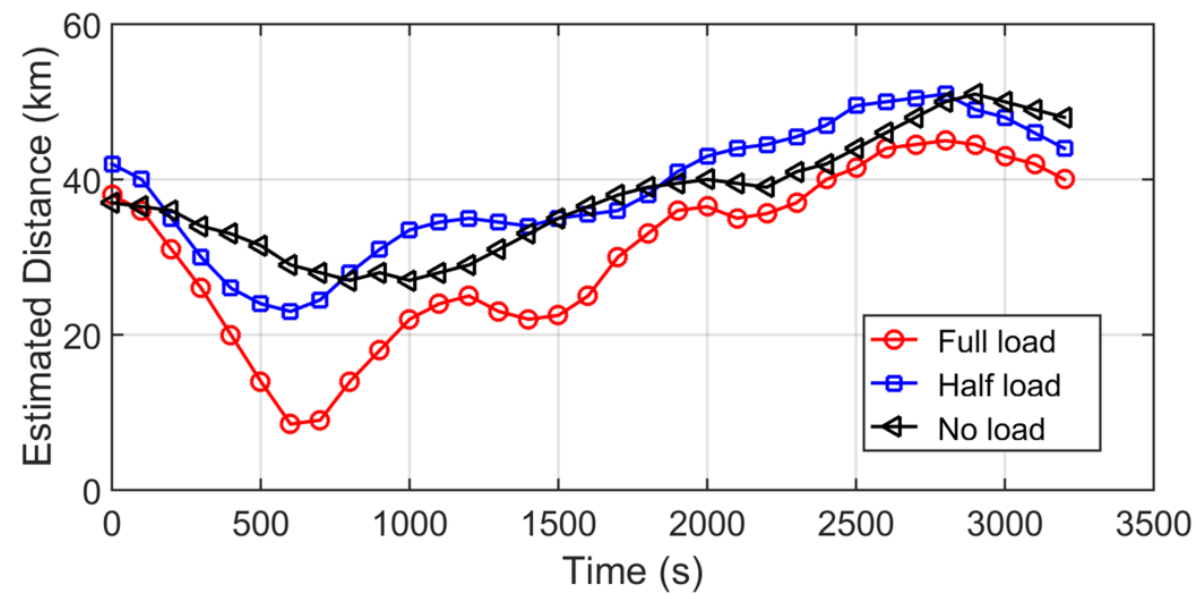

Figure 15 Output of FCM under 3 different loading conditions of EV from A to G.

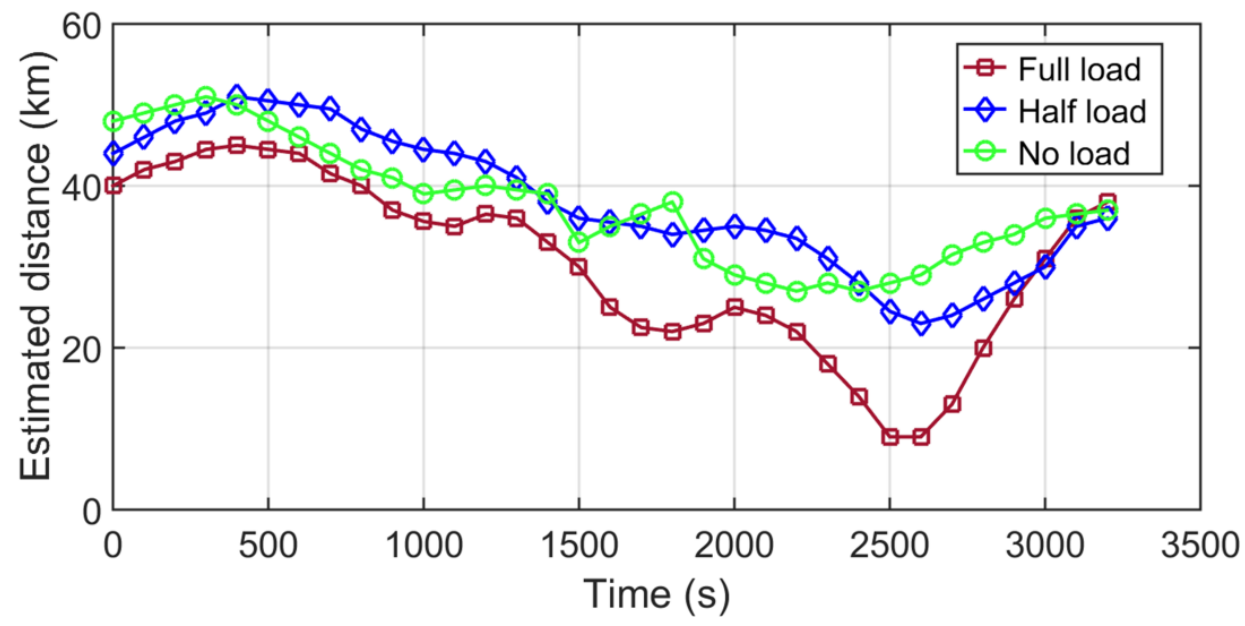

Figure 16 Output of FCM under 3 different loading conditions of EV from G to A.

\section{Comparison results}

The important feature of this study is to estimate the drive distance of EV is carried out using FCM. This approach avoids complex mathematical calculation and produces fast solutions. From the above literature, various other factors such as traffic conditions [33], environmental conditions, the external force acting on EV, and road type [34] directly affect the performance of EV. Variations in the above parameters directly reflect in the consumption of power. The parameter which affects the drive distance estimation was defined in the input parameter of the FCM design. Based on the theoretical values given in Table 5, EV can travel 14 times of the test route $(2.1 \mathrm{~km})$ under no-load conditions. Yet, the actual test result shows that the EV can travel 11 times only. This dissimilarity is getting due to the assumptions made (neglecting environmental conditions and constant speed of EV). Similarly, the $2^{\text {nd }}$ case (half load) with a $2 \%$ slope is listed in Table 5. However, the test route was completed 8 times based on the result given in Table 5. According to the result of the $3^{\text {rd }}$ case given in Table 5, the EV travel 6 times. These results show that the drive distance decreases when the load of the EV increases.

The environmental factor, the wind force also not considered while estimating the drive distance and power consumption of EV (Table 2). The wind speed of $5 \mathrm{~km} / \mathrm{h}$ was taken in this study based on [35]. When the wind speed was included in the drive distance estimation, it is observed that the drive distance is less than 1 turn from the total under full-load conditions. From the previous results, the average acceleration of the EV was found that 0.1 . The range of EV is 2.5 laps if the acceleration is defined as 0.1 under full-load conditions. The error rate of 4.8 and $20 \%$ are getting under no-load and full-load conditions. Wind speed and acceleration are important factors to reduce the error in drive 
distance estimation. When these parameters are included in drive distance estimation the accuracy of the model will increase.

\section{Conclusions}

The fuzzy classification method was utilized in this study to predict the EV's driving distance for the specified test route. The EV's driving distance was calculated using vehicle and battery characteristics. A CAN protocol was used to communicate the subsystems which are present in the vehicle and the FCM estimates the drive distance and informs the driver. The test was carried out under 3 different test conditions such as no-load, half-load, and full-load. The result showed that the battery SoC and power consumed by the vehicle directly affect the system output. Additionally, the slope of the road also affects the drive distance of the vehicle. Due to the slope up to ttest point $\mathrm{C}$ the battery voltage was decreased and the power consumed by the vehicle was increased. On the other hand, the vehicle going downhill from test point $\mathrm{D}$ to the end, the battery voltage was normal and the power consumption was decreased. Under the suggested range estimation's no-load and full-load situations, the error rate was 4.8 and $20 \%$, respectively. Road types, driver profiles, and environmental considerations can be added to the parameters impacting the EV's drive distance calculation to improve accuracy.

\section{References}

[1] E Guerra. Electric vehicles, air pollution, and the motorcycle city: A stated preference survey of consumers' willingness to adopt electric motorcycles in Solo, Indonesia. Transp. Res. D Transp. Environ. 2019; 68, 52-64.

[2] RA Hanifah, SF Toha, NHHM Hanif and NA Kamisan. Electric motorcycle modeling for speed tracking and range travelled estimation. IEEE Access 2019; 7, 26821-9.

[3] K Sarrafan, KM Muttaqi, D Sutanto and GE Town. A real-time range indicator for EVs using webbased environmental data and sensorless estimation of regenerative braking power. IEEE Trans. Veh. Technol. 2018; 67, 4743-56.

[4] S Kaya, N Kilic, T Kocak and C Gungor. A battery-friendly data acquisition model for vehicular speed estimation. Comput. Electr. Eng. 2016; 50, 79-90.

[5] J Bi, Y Wang, S Shao and Y Cheng. Residual range estimation for battery electric vehicle based on radial basis function neural network. Measurement 2018; 128, 197-203.

[6] J Bi, Y Wang, Q Sai and C Ding. Estimating remaining driving range of battery electric vehicles based on real-world data: A case study of Beijing, China. Energy 2019; 169, 833-43.

[7] C Chellaiah, K Thangamani, PG Subin, P Rathinakumar and P Muthukrishnan. Design of a fuel free electric vehicle. In: VV Das and Y Chaba (Eds.). Mobile communication and power engineering. Springer, Berlin, Heidelberg, 2013, p. 296.

[8] C Chellaswamy, L Balaji and T Kaliraja. Renewable energy based automatic recharging mechanism for full electric vehicle. Eng. Sci. Technol. Int. J. 2020; 23, 555-64.

[9] C Chellaswamy. Performance analysis of a wind duct and SOC estimation for pure electric vehicle charging. Int. J. Control Theory Appl. 2016; 9, 27-43.

[10] N Chang, MA Faruque, Z Shao, CJ Xue, Y Chen and D Baek. Survey of low-power electric vehicles: A design automation perspective. IEEE Des. Test 2018; 35, 44-70.

[11] R Basso, B Kulcsar, B Egardt, P Lindroth and I Sanchez-Diaz. Energy consumption estimation integrated into the electric vehicle routing problem. Transp. Res. D Transp. Environ. 2019; 69, 14167.

[12] HA Yavasoglu, YE Tetik and K Gokce. Implementation of machine learning based real time range estimation method without destination knowledge for BEVs. Energy 2019; 172, 1179-86.

[13] C Pan, W Dai, L Chen, L Chen and L Wang. Driving range estimation for electric vehicles based on driving condition identification and forecast. AIP Adv. 2017; 7, 105206.

[14] N Daina, A Sivakumar and JW Polak. Modelling electric vehicles use: A survey on the methods. Renew. Sustain. Energy Rev. 2017; 68, 447-60.

[15] GD Nunzio and L Thibault. Energy-optimal driving range prediction for electric vehicles. In: Proceedings of the IEEE Intelligent Vehicles Symposium, Los Angeles, CA, USA. 2017, p. 160813.

[16] X Qi, G Wu, K Boriboonsomsin and MJ Barth. Data-driven decomposition analysis and estimation of link-level electric vehicle energy consumption under real-world traffic conditions. Transp. Res. D Transp. Environ. 2018; 64, 36-52. 
[17] F Duran, S Ceven and R Bayir. Drive mode estimation for electric vehicles via fuzzy logic. In: Proceedings of the $22^{\text {nd }}$ International Conference Electronics, Palanga, Lithuania. 2018, p. 62-7.

[18] J Hong, S Park and N Chang. Accurate remaining range estimation for electric vehicles. In: Proceedings of the $21^{\text {st }}$ Asia and South Pacific Design Automation Conference, Macao, China. 2016, p. 781-6.

[19] V Gass, J Schmidt and E Schmid. Analysis of alternative policy instruments to promote electric vehicles in Austria. Renew. Energy 2014; 61, 96-101.

[20] VR Tannahill, D Sutanto, KM Muttaqi and MA Masrur. A future vision for reduction of range anxiety by using an improved state of charge estimation algorithm for electric vehicle batteries implemented with low-cost microcontrollers. IET Electr. Syst. Transp. 2015; 5, 24-32.

[21] P Boyraz and D Dogan. Intelligent traction control in electric vehicles using an acoustic approach for online estimation of road-tire friction. In: Proceedings of the IEEE Intelligent Vehicles Symposium, Gold Coast, QLD, Australia. 2013, p. 1336-43.

[22] M Dawei, Z Yu, Z Meilan and $\mathrm{N}$ Risha. Intelligent fuzzy energy management research for a uniaxial parallel hybrid electric vehicle. Comput. Electr. Eng. 2017; 58, 447-64.

[23] F Kong, L Zhang, J Zeng and Y Zhang. Automatic measurement and control system for vehicle ECU based on CAN bus. In: Proceedings of the IEEE International Conference on Automation and Logistics, Jinan, China. 2007, p. 964-8.

[24] L Wolfhard. CAN system engineering: From theory to practical applications. Springer-Verlag London, London, 2013.

[25] X Wang, W Yao and G Shi. A control system of electric vehicle based on CAN bus. In: Proceedings of the International Conference on Advanced Mechatronic Systems, Zhengzhou, China. 2011, p. $580-2$.

[26] E Bassi, F Benzi, L Almeida and T Nolte. Powerline communication in electric vehicles. In: Proceedings of the IEEE International Electric Machines and Drives Conference, Miami, FL, USA. 2009, p. 1749-53.

[27] Q Huang, J Li and Y Chen. Control of electric vehicle. Intech Open, 2012.

[28] C Chellaswamy, JJ Durgadevi and S Srinivasan. An intelligent hand gesture recognition system using fuzzy logic. In: Proceedings of the IET International Conference on Sustainable Energy and Intelligent Systems, Chennai, India. 2013, p. 326-32.

[29] M Dawei, Z Yu, Z Meilan and N Risha. Intelligent fuzzy energy management research for a uniaxial parallel hybrid electric vehicle. Comput. Electr. Eng. 2017; 58, 447-64.

[30] S Çeven and R Bayir. Implementation of fuzzy logic based speed control of brushless direct current motors via industrial PC. Int. J. Intell. Syst. Appl. Eng. 2016; 4, 146-52.

[31] S Tariq, S Lee, HK Kim and SS Woo. CAN-ADF: The controller area network attack detection framework. Comput. Secur. 2020; 94, 101857.

[32] F Kong, L Zhang, J Zeng and Y Zhang. Automatic measurement and control system for vehicle ECU based on CAN bus. In: Proceedings of the IEEE International Conference on Automation and Logistics, Jinan, China. 2007, p. 964-8.

[33] B Luin, S Petelin and FA Mansour. Modeling the impact of road network configuration on vehicle energy consumption. Energy 2017; 137, 260-71.

[34] X Yuan, C Zhang, G Hong, X Huang and L Li. Method for evaluating the real-world driving energy consumptions of electric vehicles. Energy 2017; 141, 1955-68.

[35] Meteorological data, Available at: http://www.imdchennai.gov.in, accessed April 2021. 